\title{
Making Rational Decisions in N-by-N Negotiation Games with a Trusted Third Party
}

\author{
Shih-Hung Wu and Von-Wun Soo \\ Department of Computer Science \\ National Tsing Hua University \\ Hsin-Chu City, 30043, Taiwan, R.O.C \\ shwu@cs.nthu.edu.tw, soo@cs.nthu.edu.tw
}

\begin{abstract}
The optimal decision for an agent to make at a given game situation often depends on the decisions that other agents make at the same time. Rational agents will try to find a stable equilibrium before taking an action. Rational agents can use the negotiation mechanism to reach the equilibrium. In the previous work, we proposed the communication actions of paying guarantee or compensation to convince or persuade other agents and a negotiation protocol for agents to communicate with a trusted third party. In this paper, we extend the negotiation mechanism to deal with n-by-n games and justify its optimality with the underlying assumptions. During the negotiation process, each agent makes suggestions on how they can reach equilibrium while maximizing its own payoff. The mechanism can deal with all the game situations and find an acceptable equilibrium that gives optimal payoffs.
\end{abstract}

\section{Introduction}

In a multi-agent community, the result of a certain action of an agent may depend on how other agents act. Therefore, each agent must model other agents' decision in order to find the best strategy to get the optimal outcome. Game theory provides a way to model and reason about this situation. A game matrix may represent each different outcome for all combination of strategies. According to the game theory, there are games that give a unique stable equilibrium, but also some that gives no stable equilibrium at all. Besides, multiple equilibria and prisoner's dilemma are two other well known difficult game situations. If the games allow negotiation, the negotiation protocol may help agents to solve the difficult games by changing the game matrix. If the game has no stable equilibrium point, agents should create one, beside the payoffs of the equilibrium should be acceptable. Reaching an agreement on certain equilibrium point is very important, since the agents may not always keep the commitment, changing the game matrix is a way to enforce the commitment. The stable equilibrium is often known as Nash equilibrium, therefore in the following text of the paper we treat both terms equivalently. In difficult game situations, agents should try to negotiate and change the original difficult game situation into a new game situation, in which an acceptable Nash equilibrium exists. For example, the game matrix in Fig. 1 (a) is a game matrix without Nash equilibrium and the game matrix in Fig. 1 (b) is a game matrix with multiple Nash equilibria. Agents in these 


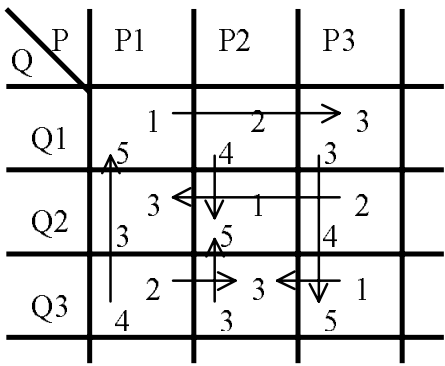

(a)

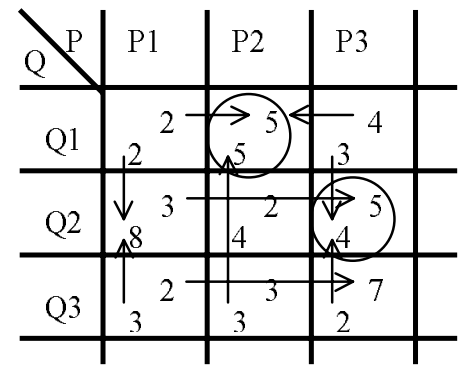

(b)

Fig. 1. The n-by-n game matrix that models a two-agent decision-making situation where each agent has three strategies. (a) The game matrix without Nash equilibrium. (b) The game matrix with multiple Nash equilibria

games need to have some negotiation mechanisms to coordinate in order to reach a stable equilibrium point that every one agrees. Traditional game theory assumes that there is no communication and both agents are mutual rational, and then they can find a mixed strategy Nash equilibrium. In this paper, we will show how the negotiation mechanism can help the agents to find a pure strategy that gives no fewer payoffs than the mixed strategy.

In the previous work [26][28], we proposed a negotiation protocol that involved a trusted third party. The mechanism can deal all the three difficult game situations in 2-by-2 games: no equilibrium, multiple equilibria and Prisoner's dilemma games. The mechanism is based on some assumptions. We should justify these assumptions are rational, so that agents that adopt the are rational. In this paper, we explore the result of negotiation in different n-by-n game situations. An n-by-n game can model more strategies for each agent, some high level strategy such as "not to play the game", "delay the decision" can also modeled into one game. This force the agent must do a rational decision at this time. This is quit different from the 2-by-2 game.

Multi-agent coordination is necessary for a multi-agent community to prevent an anarchy or chaos [5][8][13][21]. Negotiation is a way to coordinate rational agents under a game theoretical deal-making mechanism [7][17][18] [22]. Game theory can be used to guide the negotiation [16][29][30][31]. The underlying assumption is that agents model each other as rational agents and making decision base on the game theory [1][2][9][20][23]. Leveled commitment is a way to bind the commitment in multi-agent contracting, which allows punishment for the de-commitment in the future [19]. If there are different types of agents, a recursive modeling method may help in decision-making [4][6][24][25]. Issues related to uncertain games are usually not well addressed, fuzzy theory is a way to deal with uncertainty in a fuzzy game [27].

In section 2, we give the definition of negotiation games and the negotiation mechanisms. In section 3, we apply the negotiation in n-by-n games. In section 4, we show a scenario of negotiation to change equilibrium point in an n-by-n game. In section 5, we discuss on the result and in section 6 we make some conclusion. 


\section{Negotiation Games}

\subsection{The Negotiation Game}

A negotiation game is a traditional game with a negotiation mechanism. Agents in the game can analyze the game and try to find out equilibrium before taking any real action. If no acceptable equilibrium can be found in the original game, the agents will try to create one by the negotiation mechanism.

\section{Definition (A Negotiation Game)}

A one-shot negotiation game can be defined as a tuple $\langle A, S, P, N\rangle$. Where $A=\{P, Q\}$ is a set of agent, $\mathrm{S}$ is the set of strategy set for each of agent in $A, S=\left\{S_{P}, S_{Q}\right\}, S_{P}=\left\{P_{l}\right.$, $\left.\left.P_{2}, \ldots, P_{n}\right\}, S_{Q}=\left\{Q_{l}, Q_{2}, \ldots, Q_{n}\right\}\right\}, P_{P}: S_{P} \times S_{Q} \rightarrow R, P_{Q}: S_{P} \times S_{Q} \rightarrow R$ is the payoff functions that maps each combination of strategies to a payoff value for each agent and $N$ is a negotiation mechanism. The payoff functions of each agent on each combination of strategies form a game matrix. Agents may use the negotiation mechanism to alter the game matrix and find an acceptable equilibrium.

The negotiation mechanism is an external part of the game, under different situation, the mechanism must be reconstruct. We proposed a mechanism that included two communication actions and a trusted third party. The two communication actions, asking guarantee and offering compensation, provide a way to trade payoffs and therefore change the game matrix from a difficult game into a desirable game.

\subsection{The Role of the Trusted Third Party}

The basic idea of our work is that agents may trade the payoffs for a better outcome. This is very similar to the everyday bargain. We find that the existence of a trusted third party is very important. Since we assume agents are rational, a rational agent acts for its own interest only and is an expected payoff maximizer. There are game situations that can not be improved without a trusted third party. For example, in a prisoner's dilemma game, only the communication between the two agents can help to escape the dilemma. Even the agents tell each other that they will play the cooperative strategy, since each agent will try to maximize the payoff, the agents will play the defect strategy for each agent's best payoff.

In real world, the trusted third is necessary in many trade processes. If a trade process can not be finished at once and there is a possibility to play defect strategy, then there is a need of the trusted third party. For example, banks often play the role as the trusted third party in the selling and buying process of the estate. The trusted third party plays a role in the trade but the trusted third party is neither the seller nor the buyer. The trusted third can ensure the commitment of both agents to a trade with a domain independent manner. 


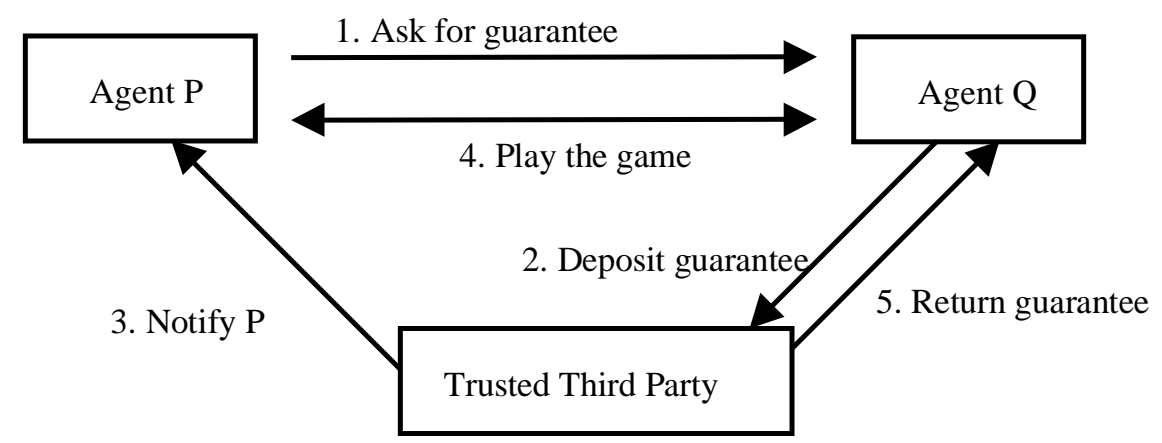

(a) The asking for guarantee communication action

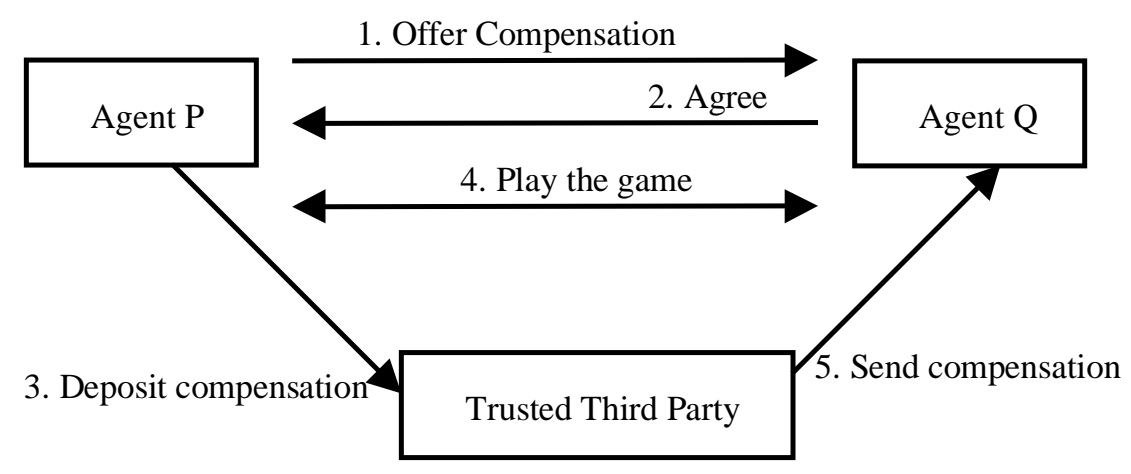

(b) The offing compensation communication action

Fig. 2. The asking for guarantee (a) and the offing compensation (b) communication actions are used together with a trusted third party in negotiation.

In our work, the trusted third party provides additional help for the enforcement of commitment. In non-cooperative games, difficult game situations can provide a selfenforcement of making a cooperative decision. We use the communication actions to alter the difficult game situations into better game situations, and this mechanism can not work without a trusted party. 


\subsection{The Guarantee, Compensation Communication Actions}

In our previous work, we defined two communication actions that can alter the game matrix, so that the game can be changed from a difficult game into one with an acceptable stable equilibrium. The two communication actions of asking guarantee and offering compensation that can help to coordinate rational agents [26][28]. The mechanism involves a trusted third party.

The guarantee communication action is a way to prevent an agent from playing a strategy that will lead to a worse result for another agent. As in Fig. 2 (a), if a strategy S1 of agent Q may lead to a less payoff result for the agent P, then the agent $\mathrm{P}$ may ask agent $\mathrm{Q}$ to deposit a guarantee $g$ at the trusted third party. The guarantee forbids agent Q to play S1. If agent Q agrees and deposits the guarantee at the trusted third party, then the trusted third will see if agent keep the commitment or not to return the guarantee or not. If agent Q plays S1, the guarantee $g$ will not be returned. If agent Q keeps the commitment, the guarantee will be returned. Therefore, if agent deposits the guarantee, we can say that the game matrix for agent $\mathrm{Q}$ is changed and the payoffs associated to the strategy S1 decrease $g$. The guarantee communication action in the n-by-n game is different from that in 2-by-2 games. In a 2-by-2 game, the guarantee for playing one strategy implies not to play another strategy. In the n-by-n game, however, the guarantee of playing a certain strategy must define in terms of not playing many other strategies. This is called the multiple forbidden property. This multiple forbidden property can be achieved by indicating the list of forbidden strategies.

Compensation communication action, on the other hand, is used to persuade an agent to play certain strategy that can lead to a desirable state. As in Fig. 2 (b), if a strategy S2 of agent Q may lead to a better payoff for the agent P, then the agent $\mathrm{P}$ may offer some compensation $c$ to persuade the agent $\mathrm{Q}$ for playing strategy $\mathrm{S} 2$. The compensation is deposited at the trusted third party temporally. The compensation will be sent to the agent $\mathrm{Q}$ if the agent $\mathrm{Q}$ does play the asked strategy S2. If agent $\mathrm{Q}$ accepts the offer, we can say the game matrix is changed. The payoffs associated to $\mathrm{S} 2$ for agent $\mathrm{P}$ decreases $c$ and the payoffs associated to $\mathrm{S} 2$ for agent $\mathrm{Q}$ increases $c$. With this negotiation protocol, we showed that rational agents can reach a Paretoefficient and Nash equilibrium in all 2-by-2 games. There is a constraint called the proper quantum principle on the minimal amount of compensation that ensures the negotiation will end within finite time.

Proper quantum principle: The minimal amount of the compensation is called a quantum. Since the basic quantum of payoff may not exist in general cases, any small but significant enough amount can be accepted at the first time. This principle implies that the next compensation should not be less than the amount that another agent previously offered. This principle is necessary to prevent that one agent may offer so small compensation that causes a lengthy negotiation process.

\subsection{The General Negotiation Protocol}

The protocol is symmetric, one agent makes a suggestion then the other agent accepts it or makes another counter suggestion. There could be many different ways to make a 
suggestion and different criteria of whether to accept a suggestion or not. We will give our approach and discuss later. The general protocol of the negotiation is:

Procedure: Negotiation Protocol

Input: the game matrix without an acceptable stable equilibrium

Output: a game matrix with an acceptable stable equilibrium

Step 1. Construct the game matrix

Then enter the making suggestion, accepting or making counter suggestion loop

Step 2. Make a suggestion to form an acceptable equilibrium point using asking guarantee and/or offering compensation communication actions based on the mutual rational assumption.

Step 3. Another agent will decide whether to accept it or not under some criteria.

Step 4. If there are better suggestions then go to step 2.

Step 5. If there is no better suggestion then accept the last suggestion.

\section{End of the Protocol}

Different criteria for the agents to make, accept, reject suggestion may cause different result of the negotiation. Here we provide a set of rules to make suggestion and decide whether to reject or accept a suggestion. First, agent form a suggestion by picking up the combination of strategies that gives the highest total payoffs. The suggestion includes the associated guarantee or compensation actions that will make this state a Nash equilibrium point. When agents offer the compensation, they follow the assumption of proper quantum principle. Second, the agent accepts only the suggestion that gives higher payoff then its own suggestion and rejects other suggestion. These rules imply that the negotiated result must be Nash equilibrium and which gives the highest total payoffs while each agent seeking its own interesting. In our previous work, we have proved that these rules can escape the prisoner's dilemma, end up with the same payoffs in multiple Nash equilibrium games, no matter which agent initiate the negotiation [28]. In this paper, we don't limit the agent to these rules, since different rules that take different preference in to consideration may have different result. For example, if finish the negotiation as soon as possible when seeking a stable and acceptable equilibrium point is the most important job, accept the first suggestion is a possible rule. If we allow that the two agents have different eagerness of seeking the final negotiated result, the negotiation may have different result on who initiate the negotiation.

\section{Applying the Negotiation Mechanism to N-by-N Games}

In this section we describe how the negotiation mechanism works in n-by-n games.

\subsection{Escape from the Prisoner's Dilemma in an N-by-N Game}

The prisoner's dilemma (PD) is a special game in game theory literature [14]. Since many phenomena can be modeled by the PD game [1], such as the multi-agent coordination [10][11]. In a PD game, each agent will choose its own dominant strategy but the outcome (combination of the strategies) will lead to a worst result. In a 2-by-2 prisoner's dilemma game, agents will ask for a guarantee of not playing the 
defect strategy but playing the cooperative strategy. Thus, the agents can escape the dilemma and reach a new equilibrium that gives more payoffs for both agents.

A prisoner's dilemma game involves two agents, each agent has two strategies to play: the cooperative and the defect strategies. The payoffs of the combination of (cooperative, cooperative), (cooperative, defect), (defect, cooperative), (defect, defect) are (a, a), (b, c), (c, b) and (d, d) respectively. Where c $>a>d>b$. A standard prisoner's dilemma game requires $2 \mathrm{a}>\mathrm{b}+\mathrm{c}$. And the cases in which $2 \mathrm{a}<\mathrm{b}+\mathrm{c}$ are also called prisoner's dilemma. In a prisoner's dilemma game, the agents will play the dominant strategy (defect, defect) since $c>a$ and $d>b$ and get the payoffs $(d, d)$ while bypass the better payoffs $(a, a)$.

With a trusted third party as an intermediary agent, using the guarantee communication action alone is enough for the agents to escape from a standard prisoner's dilemma in an 2-by-2 game. The agents can simply deposit the guarantee of not playing defect strategy at the trusted third party. It will reduce the payoff of playing the defect strategy significantly enough that make defect no longer a dominant strategy. Then combination of (defect, defect) strategy is no longer a Nash equilibrium point, and thus the agents will find another equilibrium eventually. After agents paying the guarantee, there could be several new Nash equilibria that will have higher payoffs than the original dilemma Nash equilibrium.

If more choices of strategies instead of two are available for each agent, the definition of a prisoner's dilemma game can be extended from a 2-by-2 game to an nby-n game.

Definition (Generalized Prisoner's dilemma game)

A generalized Prisoner's dilemma game is a game where there is only one Nash equilibrium point, but the equilibrium point is Pareto dominated by other strategy combination of the game.

For the generalized Prisoner's dilemma game, however, only the guarantee of not playing defect strategy is not enough to find an optimal decision. The compensation action is necessary. We may also add a constraint to define a generalized standard prisoner's dilemma game. The sum of the payoffs for any combination of strategies that involves the defect strategy should be less than the sum of the payoffs for some combination of strategies that involves no defect strategy. Then guarantee communication alone can help to escape from the generalized standard prisoner's dilemma.

\subsection{Choose or Form One Nash Equilibrium Point in an N-by-N game}

The technique of choosing or forming one Nash equilibrium point in 2-by-2 games can be directly applied to n-by-n games. Each agent may suggest certain combination of strategies as a candidate stable equilibrium and reject or accept other agent's suggestion. If no mutual agreement is reached, then offering the compensation to persuade each other is a way to reach a new compromise state. Each suggestion may accompany with the guarantee or compensation communication actions that ensure the suggestion is a Nash equilibrium point. Both guarantee and compensation are useful. We will show a scenario of negotiation process in an n-by-n game, where 
there is already a Nash equilibrium point, but with the help of guarantee and compensation, agents can form a new Nash equilibrium point and get higher payoffs.

\section{A Scenario of Negotiation of Changing the Nash Equilibrium in an N-by-N Game}

In this section, we show an example of using the suggestion, rejection, acceptance actions as well as the guarantee and compensation communication actions to help agents getting more payoffs in seeking Nash equilibrium. In this example, we assume that agents will suggest and accept only strategy combination that gives the highest payoff for itself. And if there is a need to persuade other agent, agents will offer the minimum required compensation. The game matrix in Fig. 3 (a), with one Nash equilibrium at strategy combination $(\mathrm{Q} 1, \mathrm{P} 3)$ and the payoff for $(\mathrm{Q}, \mathrm{P})$ is $(2,3)$ respectively. Now we show how the negotiation can alter the game and reach a new Nash equilibrium point that gives better payoffs $(3,4)$. The negotiation procedure proceeds as follows: (For the simplicity, the communications with the trusted third party for the guarantee and compensation actions are omitted).

1. Agent $\mathrm{P}$ suggests $(\mathrm{Q} 1, \mathrm{P} 3)$ since it gives the highest payoff for agent $\mathrm{P}$ and it is a Nash equilibrium point.

2. Agent $Q$ rejects the suggestion since it does not give the highest payoff for agent $\mathrm{Q}$.

3. Agent $\mathrm{Q}$ suggests $(\mathrm{Q} 2, \mathrm{P} 1)$ and asks guarantee 3.1 from $\mathrm{P}$ that $\mathrm{P}$ will not play $\mathrm{P} 2$ or $\mathrm{P} 3$. The suggestion $(\mathrm{Q} 2, \mathrm{P} 1)$ gives the highest payoff for agent $\mathrm{Q}$, but it is not a Nash equilibrium point. A guarantee 3.1 for $\mathrm{P}$ not to play $\mathrm{P} 2, \mathrm{P} 3$ will make it a Nash equilibrium point.

4. But Agent $\mathrm{P}$ rejects the suggestion since it does not give the highest payoff for agent $\mathrm{P}$.

5. Agent $\mathrm{P}$ suggests $(\mathrm{Q} 1, \mathrm{P} 3)$ again, since it gives the highest payoff for agent $\mathrm{P}$ and it is a Nash equilibrium point.

6. Agent $Q$ rejects again.

7. Agent $\mathrm{Q}$ suggests $(\mathrm{Q} 2, \mathrm{P} 1)$ since it gives the highest payoff for agent $\mathrm{Q}$, and offers a compensation 3.1 for $\mathrm{P}$ to play $\mathrm{P} 1$ to persuade agent $\mathrm{P}$ for leaving $(\mathrm{Q} 1, \mathrm{P} 3)$ and playing (Q2, P1).

8. Agent $\mathrm{P}$ accepts the compensation since the new suggestion gives higher payoff than he could get by its previous suggestion as indicated in Fig. 3 (b).

9. Agent $\mathrm{P}$ finds that there is a better strategy combination $(\mathrm{Q} 1, \mathrm{P} 1)$ after accepting compensation from agent $\mathrm{Q}$. Therefore, agent $\mathrm{P}$ suggests $(\mathrm{Q} 1, \mathrm{P} 1)$ and offers a compensation 1.1 for $\mathrm{Q}$ to play $\mathrm{Q} 1$ since it gives the highest payoff for agent $\mathrm{P}$ and can persuade agent $\mathrm{Q}$ to leave $(\mathrm{Q} 2, \mathrm{P} 1)$ and play $(\mathrm{Q} 1, \mathrm{P} 1)$.

10. Agent $\mathrm{Q}$ accepts the compensation and makes no more suggestion since the other strategy combinations will be dominated by the $(\mathrm{Q} 1, \mathrm{P} 1)$ if agent $\mathrm{Q}$ tries to offer some compensation to persuade agent $\mathrm{P}$ as shown in Fig. 3 (c). 


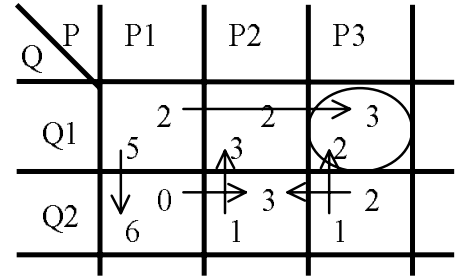

(a)

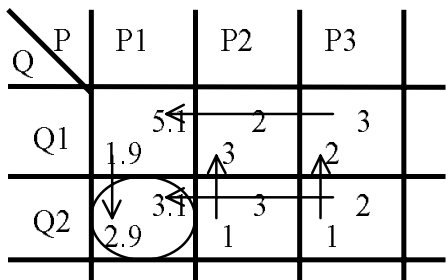

(b)

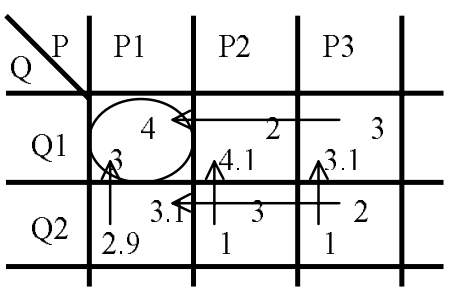

(c)

Fig. 3. An n-by-n game matrix that models a two-agent decision-making situation with one Nash equilibrium where (a) is the original game matrix, (b) is the game matrix after $Q$ offering a compensation of 3.1 for $\mathrm{P}$ to play $\mathrm{P} 1$, (c) is the game matrix after $\mathrm{P}$ accepting the suggestion and offering a compensation of 1.1 for Q to play Q1.

11. Both agents reach a compromise that is a new Nash equilibrium point and get higher payoffs $(3,4)$.

The result after negotiation is: agent $\mathrm{P}$ pay compensation of 1.1 for $\mathrm{Q}$ to play $\mathrm{Q} 1$ while agent $\mathrm{Q}$ pay compensation 3.1 for $\mathrm{P}$ to play $\mathrm{P}$ 1. Agent $\mathrm{P}$ and agent $\mathrm{Q}$ will eventually end up with getting payoffs 3 and 4 respectively. At each step, the agent will suggest a strategy combination that gives a better payoff for itself. Each suggestion must associate with communication actions that will lead to a Nash equilibrium point. As shown in this example, at each step, the suggestion will always lead to Nash equilibrium, but the payoffs change in different Nash equilibrium points. The final negotiated Nash equilibrium point gives more payoffs for each agent than the payoff of the original Nash equilibrium point. The agent always suggests the strategy combination that gives the highest payoff for itself (a rational agent). The agent accepts the other agent's suggestion only when it gives more payoffs than the previous suggestion made by itself. The suggestion always goes with either an asking guarantee or offering compensation action that will make the suggestion a Nash equilibrium point. 


\section{Discussion}

\subsection{The Need of Negotiation}

In a negotiation game, agents will try to reach an equilibrium point before they play the game. In different game situations, agents need to apply different negotiation mechanisms to achieve the goal of finding an equilibrium point. The benefit of negotiation to escape from prisoner's dilemma is obvious. Here we discuss the other two difficult game situations with no or multiple Nash equilibria.

In no or multiple Nash equilibria games, if the agents could not reach a compromise game situation, they can only play the mixed strategy defined by Nash in the game theory. Each agent has a vector of probabilities associated to every strategy that the agent may play. Nash has proofed the existence of the mixed strategy Nash equilibrium for each game. The vector can also help to calculate the expected payoff. Since the expected payoff is a weighted sum of the payoffs in the possible outcomes, unless in a zero-sum or constant game, the summation of both agents' expected payoffs must be less than the highest summation in the game. Therefore, there is a driving force for the agents to reach the state that gives the highest summation of payoffs for both, and to offer some compensation so that each may get more payoff than playing the mixed strategy. In a zero-sum or constant-sum game, the negotiation mechanism can also help the agent to reach a Nash equilibrium point in which each agent gets the same payoff as the agents play the mixed strategies.

For example, consider the constant-sum game matrix in Fig. 1 (a). The expected payoff of the game $(4,2)$ can be deduced from the symmetric game matrix. The bestnegotiated payoff is also $(4,2)$. It could be for the agent $\mathrm{Q}$ who suggests $(\mathrm{Q} 1, \mathrm{P} 1)$ and offers compensation 1 and asking a guarantee 1.1 for agent $\mathrm{P}$ to play P3. Or it could be for the agent $\mathrm{P}$ who suggests (Q1, P3) and offers compensation 1 and asks a guarantee 1.1 for agent Q to play Q1. The two ways will lead to two different Nash equilibrium points, but the final payoffs for each agent remains the same.

In another example, consider the matrix in Fig. 1 (b) where there are multiple Nash equilibrium points. Any mixed strategy can not help the agents get better payoffs than the negotiated payoffs $(6,5)$. The final state of the negotiation is that the agent $Q$ suggests $(\mathrm{Q} 2, \mathrm{P} 1)$ while offering compensation 2 and asking a guarantee 0.1 for agent $\mathrm{P}$ to play $\mathrm{P} 1$. These actions make $(\mathrm{Q} 2, \mathrm{P} 1)$ a Nash equilibrium point and each agent will get a higher payoff than that of the original Nash equilibrium points.

\subsection{The Suggest, Accept and Reject Actions}

The suggesting actions, accepting actions and rejecting actions can be very versatile in actual human negotiation tactics. We just give one way that is feasible for software agents. Agents make suggestions that belong to the Pareto efficient set and reject other suggestions. Agents accept only the suggestions that give the highest payoff after taking the compensation and guarantee into account. We can give some important result under the assumption of proper quantum principle. But the proper 
quantum principle is not always a fair assumption. If the agents have the right to offering different scale of compensation, the result will be quite different. There could be a need of new consideration and new communication actions. For example, taking the loss of not reaching a compromise into account, adding threat or stand still as new actions.

\subsection{The Underlying Assumptions}

To apply the negotiation game as a coordination tool, there are several assumptions that make the negotiation game valid.

1. The game matrix can be constructed.

2. The agents assume mutual rationality.

3. The transfer of payoff is possible.

4. The trusted third party exists and can take the communication actions.

5. The results of the actions that agents act are observable by all the agents.

In addition to the five assumptions above, it requires also constraints to help the mechanism work. First is the proper compensation quantum principle as we mentioned. We argued that the offering of the compensation must be fair. That is, an agent should not make an offer less than that of other agent. This principle prevents the situation that only one agent is making compromise while the other agent is staying no reaction. This principle is arguable. In reality, one agent may stand still if it doesn't care about failing to reach a Nash equilibrium point or the agent has no obligation to play the game. Second is the notion of treating payoffs as the same if the difference is small enough. This can help agents to reach the equilibrium faster without calculating compensation many times.

\subsection{The Difficulty of Applying the Negotiation Game to N-person Games}

So far we discuss only 2-person games with a trusted third party. We do not apply the mechanism to n-person games. The difficulty comes from two folds. First it is hard to construct the game matrix considering the complexity. The number of combination of strategies increases in an exponential way. For the game remain tractable, $\mathrm{n}$ must be a relative small number. Second, it must redefine the concept of compensation. In two person games, the compensation is offered by one agent and received by another. In n-person games, the compensation offered by one agent may be sent to one or many agents under different circumstances. The distribution of compensation can be divided into many ways.

\section{Conclusion}

The purpose of negotiation game is to ensure the binding of commitment so that the agents can get more payoffs. Extend the negotiation game into n-by-n can model the decision "not to play the game" as a strategy into the game. This force the agent must play the game. The "must play" condition forces the agent must negotiate for a better result. Since we assume agents are rational, they will try to reach Nash 
equilibrium while seeking for a higher payoff. In this paper, we show that the negotiation game can be applied to n-by-n games. The agents can escape form the generalized prisoner's dilemma game, and get more payoffs in several game situations. The payoff each agent can get in a negotiated equilibrium is, unless in a zero-sum or constant-sum game, more than that each agent may get in mixed strategy equilibrium. In a zero-sum or constant-sum game, the agents get the same payoffs as the mixed strategy. However, it is hard to apply the mechanism on n-person game. We left it as the future work.

\section{Acknowledgment}

The work is financially supported by National Science Council of Taiwan, Republic of China, under the grant number NSC88-2213-E-007-057.

\section{Reference}

1. Axelrod, R.: The Evolution of Cooperation, Basic Books Inc., New York, 1984.

2. Brafman, R.I. and Tennenholtz, M.: Modeling Agents as Qualitative Decision Makers, Artificial Intelligence, Vol.94, pp217-268, 1997.

3. Brams, S.J.: Theory of Moves, American Scientist, Vol. 81, pp.562-570, 1993.

4. Durfee, E.H., Lee, J. and Gmytrasiewicz, P.J.: Overeager Reciprocal Rationality and Mixed Strategy Equilibria, In Proceedings of the Eleventh National Conference on Artificial Intelligence (AAAI-93), pp.225-230, 1993.

5. Genesereth, M.R., Ginsberg, M.L. and Rosenschein, J.S.: Cooperation without Communication, In Proceedings of the National Conference on Artificial Intelligence (AAAI-86), pp.51-57, Philadelphia, Pennsylvania, 1986.

6. Gmytrasiewicz, P.J., Durfee, E.H. and Wehe, D.K.: The Utility of Communication in Coordinating Intelligent Agents, in Proceedings of the ninth national conference on Artificial Intelligence (AAAI-91), pp.166-172, 1991.

7. Haynes, T. and Sen, S.: Satisfying User Preferences while Negotiating Meetings, in Proceeding of the Second International Conference on Multi-Agent Systems (ICMAS-96), 1996.

8. Jennings, N.R.: Controlling Cooperative Problem Solving in Industrial Multi-agent System Using Joint Intentions, Artificial Intelligence 75. 1995.

9. Koller, D. and Pfeffer, A.: Representations and Solutions for Game-theoretic Problems, Artificial Intelligence, Vol.94, pp167-215, 1997.

10. Matsubara, S. and Yokoo, M.: Cooperative Behavior in an Iterated Game with a Change of the Playoff Value, ICMAS-96, pp.204-211, 1996.

11. Mor, Y. and Rosenschein, J.S.: Time and the Prisoner's Dilemma, In Proceeding of the First International Conference of Multi-Agent System (ICMAS-95), pp.276-282, 1995.

12. Nash, J.F.: Non-cooperative games, Ann. of Math. 54, pp.286-295, 1951.

13. Nwana, H.S., Lee, L.C. and Jennings, N.R.: Coordination in Software Agent Systems, BT Tech. J., 14(4), 1996.

14. Rasmusen, E.: Games and Information: An Introduction to Game Theory, Basil Blackwell, Oxford, 1989.

15. Rosenschein, J.S. and Genesereth, M.R.: Deals among Rational Agents, in Proceedings of the ninth international conference on Artificial Intelligence (IJCAI-85), pp.91-99. 1985.

16. Rosenschein, J.S. and Zlotkin, G.: Rules of Encounter, MIT Press, Cambridge, 1994. 
17. Sandholm, T.W. and Lesser, V.R.: Issues in Automated Negotiation and Electronic Commerce: Extending the Contract Net Framework, in Proceedings of the First International Conference on Multi-Agent Systems (ICMAS-95), pp.328-335, MIT Press, 1995.

18. Sandholm, T.W. and Lesser, V.R.: Equilibrium Analysis of the Possibilities of Unenforced Exchange in Multiagent Systems, Proceedings of the Fourteenth International Joint Conference on Artificial Intelligence, pp.694-701, 1995.

19. Sandholm, T.W. and Lesser, V.R.: Advantages of a leveled commitment contracting protocol, In Proceedings of the National Conference on Artificial Intelligence, pp.126-133, 1996.

20. Shehory, O. and Kraus, S.: Methods for Task Allocation via Agent Coalition Formation, Artificial Intelligence, Vol.101, pp.165-200, 1998.

21. Smith, R.G.: The Contract Net Protocol: High-level Communication and Control in a Distributed Problem Solver, IEEE Trans. on Computers, 1980, C29, (12)

22. Sycara, K.: Resolving Goal Conflicts via Negotiation, AAAI-88, pp.245-250, 1988.

23. Tennenholtz, M.: On Stable Social Laws and Qualitative Equilibria, Artificial Intelligence, Vol.102, pp.1-20, 1998.

24. Vidal, J.M. and Durfee, E.H.: Recursive Agent Modeling Using Limited Rationality, in Proceedings of the First International Conference on Multi-Agent Systems (ICMAS-95), pp. 376-383, MIT Press, 1995.

25. Vidal, J.M. and E.H. Durfee, E.H.: Using Recursive Agent Models Effectively, Proceedings on the IJCAI Workshop on Intelligent Agents II: Agent Theories, Architectures, and Languages, LNAI, Vol.1037, pp. 171-186, Springer Verlag, 19-20 August 1996.

26. Wu, S. and Soo, V.: Escape from a Prisoners' Dilemma by Communication with a Trusted Third Party, in Proceeding of the Tenth International Conference on Tools with Artificial intelligent (ICTAI'98), pp.58-65, 1998.

27. Wu, S. and Soo, V.: A Fuzzy Game Theoretic Approach to Multi-Agent Coordination, Pacific Rim international workshop of on Multi-Agent (PRIMA'98), 1998.

28. $\mathrm{Wu}, \mathrm{S}$. and Soo, V.: Game Theoretic Reasoning in Multi-agent Coordination by Negotiation with a Trusted Third Party, in Proceeding of the Third International Conference on Autonomous Agents (Agents'99), Seattle, Washington, pp.56-61, 1999.

29. Zlotkin, G. and Rosenschein, J.S.: Negotiation and Task Sharing among Autonomous Agents in Cooperative Domains, IJCAI-89, pp.912-917, 1989.

30. Zlotkin, G. and Rosenschein, J.S.: Compromise in Negotiation: Exploiting Worth Functions over States, Artificial Intelligence, Vol.84, pp.151-176, 1996.

31. Zlotkin, G. and Rosenschein, J.S.: Mechanisms for Automated Negotiation in State Oriented Domains, Journal of Artificial Intelligence Research, Vol.5, pp.163-238, 1996. 